\title{
RASTREAMENTO DE DERMATÓFITOS EM ESMALTES DE UNHA UTILIZADOS EM ESTABELECIMENTOS DE BELEZA
}

\author{
Karoline Gomes Dias Bezerra1, Igor Firmino Costa ${ }^{1}$, Egberto Santos Carmo ${ }^{2}$ \\ 1 Graduandos do Curso de Bacharelado em Farmácia, Unidade Acadêmica de Saúde, \\ Universidade Federal de Campina Grande, Cuité-PB, Brasil.
}

${ }^{3}$ Prof. da Unidade Acadêmica de Saúde, Universidade Federal de Campina Grande, Cuité, PB, Brasil.

E-mail para correspondência: Kg_karol@hotmail.com

\begin{abstract}
Resumo
O uso de cosméticos compartilhados em estabelecimentos de beleza como é o caso dos esmaltes, pode desempenhar um importante papel na disseminação de microrganismos, sejam eles patogênicos ou não. O contato direto deste tipo de cosmético com a região das unhas pode estar relacionado à transmissão de dermatófitos, fungos estes responsáveis pelo desenvolvimento de tinea unguium. Objetivou-se investigar a possível contaminação por dermatófitos, em esmaltes utilizados por manicures em seus estabelecimentos, no município de Cuité-PB. A coleta das amostras foi realizada no mês de junho de 2019, em três estabelecimentos de beleza, por meio de swabs, e colocadas em tubos de ensaio com caldo BHI (Brain Heart Infusion) e solução salina 0,9\%. Em seguida foram cultivadas em Ágar Sabouraud Dextrose acrescido de ampicilina e incubadas a $25^{\circ} \mathrm{C}$ por um período de até 15 dias. Não foi detectada a presença de dermatófitos nas nove amostras analisadas. Este resultado corrobora a eficácia do sistema de conservantes que compõem os cosméticos analisados. De todo modo, faz-se necessário desenvolver ações de conscientização e instrução dos profissionais dos estabelecimentos sobre a utilização adequada de utensílios compartilhados.
\end{abstract}

Palavras-chave: Dermatomicoses. Onicomicose. Centros de Embelezamento e Estética.

\begin{abstract}
The use of shared cosmetics such as enamels in beauty salons can play an important role in the spread of microorganisms, whether they are pathogenic or not. The direct contact of this type of cosmetic with the nails may be related to the transmission of dermatophytes, fungi that are responsible for the development of tinea unguium. The objective was to investigate the possible contamination by dermatophytes, in enamels used by manicurists in their establishments, in the municipality of Cuité-Paraíba. The collection of samples was carried out in June
\end{abstract}


2019 , in three beauty establishments, by means of swabs, and placed in test tubes with $\mathrm{BHI}$ broth (Brain Heart Infusion) and $0.9 \%$ saline solution. Then, they were grown on Sabouraud Dextrose Agar plus ampicillin and incubated at $25^{\circ} \mathrm{C}$ for a period of up to 15 days. The presence of dermatophytes was not detected in the nine samples analyzed. This result corroborates the effectiveness of the preservative system that make up the analyzed cosmetics. In any case, it is necessary to develop awareness and instruction actions by the professionals of the establishments on the proper use of shared utensils.

Keywords: Dermatomycosis. Onychomycosis. Beautification and Aesthetics Centers.

\section{INTRODUÇÃO}

Os dermatófitos são fungos queratinofílicos, representados pelos gêneros Trichophyton, Microsporum e Epidermophyton, os quais são responsáveis pelo desenvolvimento de diversas micoses cutâneas, caracterizadas por reação inflamatória a partir da fixação no extrato córneo de pele, unhas e cabelo. Dependendo da espécie, esses dermatófitos podem ser classificados em geofílicos, antropofilicos ou zoofílicos (PEIXOTO et al., 2010; BRONDANI et al., 2016; SAHHO; MAHAJAN, 2016).

As micoses cutâneas provocadas pelos dermatófitos recebem o nome de dermatofitoses ou dermatomicoses, sendo de caráter contagioso, podendo acometer diversos locais no corpo. A tinea unguium também conhecida como onicomicose é uma infecção fúngica frequente na população, principalmente acometendo as unhas dos pés, sendo responsável por $15 \%$ a $40 \%$ de todas as infecções fúngicas, tendo o Trichophyton rubrum como agente mais comum mundialmente (CHIACCHIO et al., 2013, SAHHO; MAHAJAN, 2016).

Muitos são os fatores de risco para o desenvolvimento de onicomicoses, incluindo idade, ocupação, calçados oclusivos, secagem inadequada dos pés, atividades esportivas, hiperidrose, diabetes mellitus, imunossupressão, doença vascular periférica, trauma, tinea pedis, entre outros. Clinicamente podem ser observados quadros de acantose, hiperquaratose grave e hiperpigmentação da camada basal da unha (CHIACCHIO et al., 2013, MARTíNEZ-HERRERA et al., 2018).

Por se tratar de uma doença contagiosa, objetos inanimados, como os esmaltes utilizados por manicures em Centros de Embelezamento e Estética, pode ser uma importante fonte de contaminação cruzada por dermatófitos, e apesar da grande utilização dos mesmos no cotidiano dos brasileiros, estudos sobre a segurança da aplicação desses produtos, quanto a este tipo de 
contaminação são escassos (RABUSKE; BARACY; ORZECHOWSKI, 2014; CAROBELI et al., 2019). Com base nisso, esta pesquisa visa investigar a possível contaminação por dermatófitos, em esmaltes utilizados por manicures em seus estabelecimentos, no município de Cuité-PB.

\section{MATERIAIS E MÉTODO}

\subsection{Local de trabalho}

Três estabelecimentos de beleza da cidade de Cuité/Paraíba - Brasil, categorizados como "A, B e C", além do Laboratório de Microbiologia da Unidade Acadêmica de Saúde (UAS), no Centro de Educação e Saúde (CES) em Cuité-PB, da Universidade Federal de Campina Grande (UFCG).

\subsection{Coleta das amostras}

Três esmaltes de cada salão de beleza foram avaliados durante o mês de junho de 2019. As amostras escolhidas foram os esmaltes mais utilizados e que estivessem dentro do prazo de validade, sendo eles 3 incolor, 3 de tonalidade clara e 3 da tonalidade escura de cada estabelecimento. Para cada coleta utilizou-se um swab, o qual, após entrar em contato com o esmalte, era colocado em tubos de ensaio estéreis, contendo $0,5 \mathrm{~mL}$ de solução salina $0,9 \%$ e tubos contendo $0,5 \mathrm{~mL}$ de $\mathrm{BHI}$ (Brain Heart Infusion), sendo estes levados imediatamente sob acondicionamento, em caixa térmica contendo gelo para o Laboratório de Microbiologia da UFCG - CES - UAS, onde foram processadas.

\subsection{Meios de cultura}

Para isolamento fúngico, o material coletado foi semeado na superfície de Ágar Sabouraud Dextrose preparado com ampicilina de acordo com as instruções do fabricante.

\subsection{Preparo das amostras e identificação de dermatófitos}

As amostras semeadas no meio de cultura citada e incubadas em estufa a $25^{\circ} \mathrm{C}$ por até 15 dias (Figura 1). Para possível identificação foi feito a visualização de lâmina contendo fragmento da colônia da cultura corada com azul de metileno. E, para identificação das espécies fúngicas foram utilizados 
os livros e atlas de micologia (HOOG et al., 2001; LACAZ et al, 2002; SIDRIM; ROCHA, 2010).

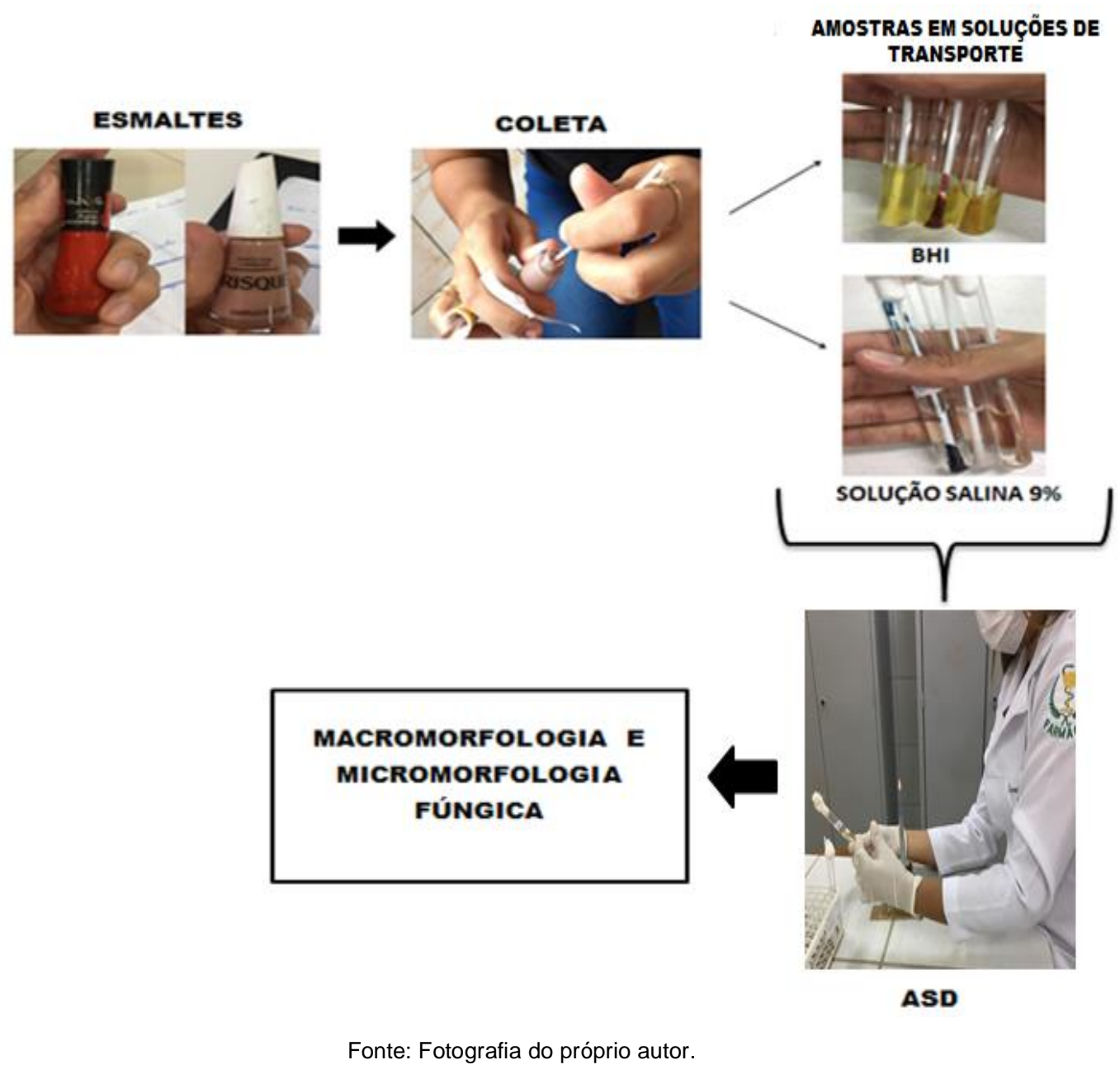

Figura 1 - Fluxograma da metodologia adotada na pesquisa de dermatófitos em esmaltes.

\section{RESULTADOS E DISCUSSÃo}

Após o período de incubação, foi possível observar que não houve crescimento fúngico para as nove amostras selecionadas e semeadas em Ágar Sabouraud Dextrose acrescido de antibacteriano (Figura 2).

O resultado negativo para todas as amostras analisadas demonstra a efetividade do sistema de conservantes nos esmaltes avaliados, condizendo com a própria definição estabelecida para esse tipo de sistema, que afirma reduzir a probabilidade de crescimento e posterior contaminação em produtos, mesmo que estes sejam frequentemente utilizados, ratificando a segurança do cosmético em questão (PEREIRA, 2011). 
Ressalta-se que este resultado atende ao que a Agência Nacional de Vigilância Sanitária (ANVISA) preconiza, quando determina que a quantidade de conservante usada em uma formulação deverá ser a mínima necessária para promover a proteção do produto, sem prejuízo para o paciente e consumidor (ANVISA, 2019).

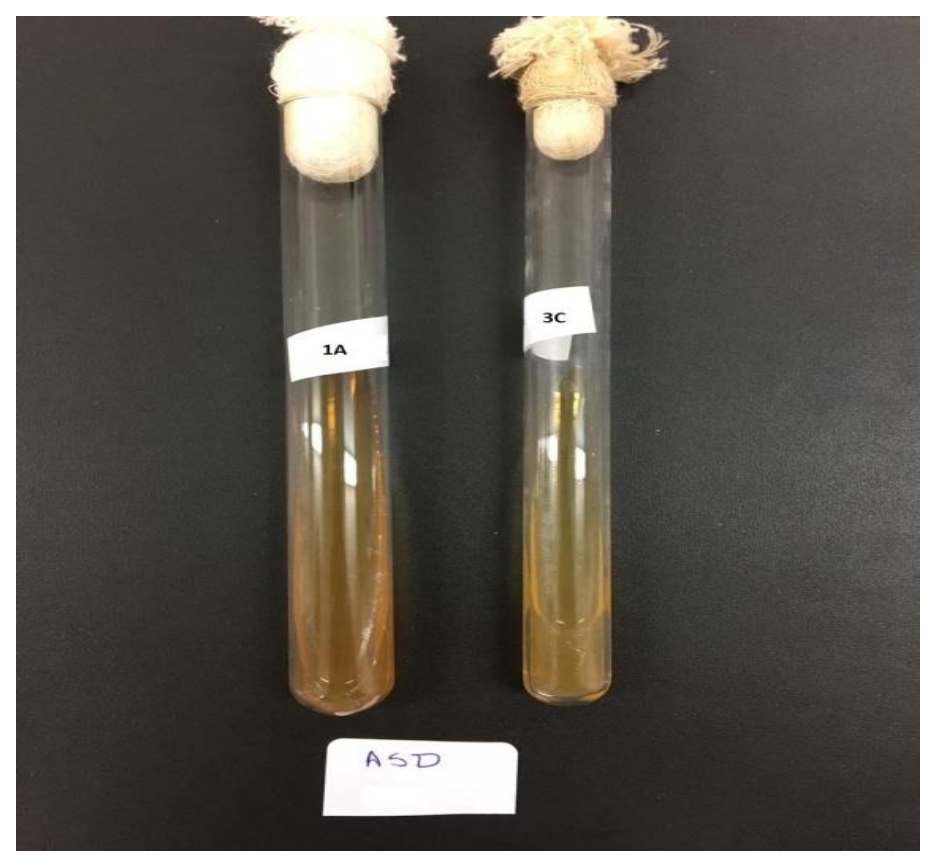

Fonte: Fotografia do próprio autor

Figura 2 - Análise macroscópica das culturas realizadas para os esmaltes 1A e 3C.

Assim, todos os esmaltes analisados apresentam em sua composição o álcool isopropílico. Segundo Reis; Braibante; Miranda (2017) os álcoois são os principais responsáveis pela conservação microbiológica de esmaltes, somado aos acetatos. O Álcool isopropílico, também denominado isopropanol é o conservante mais utilizado na composição de esmaltes, vale salientar que sua atividade contra todo tipo de microrganismo apenas é efetiva, quando em concentrações acima de 50\% na formulação (COUTINHO et al., 2017).

Os solventes que compõem o sistema de conservante presente no esmalte são considerados voláteis, o uso diário, coletivo e excessivo desses cosméticos nos sugere que a concentração destes compostos voláteis, seja menor comparando a sua concentração inicial e final do frasco (BENEDETTI, 2016). 
Em uma pesquisa realizada por Almeida (2013) no interior de São Paulo com esmaltes de unha, quatro espécies de fungos foram identificadas, Trichophyton rubrum, Trichopyton mentagrophytes, Candida albicans e Candida parapsilosis, ainda no primeiro dia de análise das culturas, o que demonstra um importante crescimento fúngico nos esmaltes analisados, sendo os dois primeiros dermatófitos que mais provocam onicomicose na população.

Destaca-se que os produtos cosméticos necessitam de uma avaliação da qualidade sanitária, mesmo que pertençam ao grupo de produtos de Grau 1, segundo a ANVISA, que define que tais produtos não apresentam comprovação inicialmente necessária e não requer informações detalhadas quanto ao seu modo de usar e suas restrições de uso (ANVISA, 2015).

Salienta-se que a atenção da transmissão de microrganismos em salões de beleza deve ser efetiva, necessitando de maior regulamentação pela legislação sanitária (GARBACCIO; OLIVEIRA, 2013).

Além de problemas relacionados ao produto cosmético, Oliveira et al. (2014) realizaram um estudo com a participação de 67 manicures e com os resultados detectaram que $48 \%$ delas, não receberam informações sobre as doenças infectocontagiosas e apenas $21 \%$ utilizavam luvas, durante os procedimentos. Com relação à esterilização dos utensílios, 58\% relataram que esterilizavam, porém os métodos não eram adequados.

Alguns estudos relatam que muitos profissionais da área de beleza não utilizam equipamento de proteção individual e os procedimentos de esterilização de materiais são inadequados, sugerindo assim a necessidade de ações de qualificação aos profissionais quanto aos aspectos da importância da saúde coletiva (GARBACCIO; OLIVEIRA, 2012; OLIVEIRA et al., 2014; OLIVEIRA et al., 2015).

\section{CONCLUSÃO}

No presente trabalho, não se observou crescimento de dermatófitos nas amostras dos esmaltes analisados nos estabelecimentos de beleza do município de Cuité-Paraíba. Apesar disso, sabendo-se da volatilidade dos solventes/conservantes empregados nos esmaltes, necessita-se de maior 
atenção pelos órgãos reguladores no que se refere ao controle de qualidade desses produtos, proporcionando maior segurança aos profissionais manicures e ao cliente no qual estes esmaltes serão utilizados, bem como outros utensílios utilizados nas unhas.

\section{REFERÊNCIAS}

ALMEIDA, Margarete Teresa Gottardo. Nail polishes: Uncommon fomites for the transmission of pathogenic fungi. Journal of Microbial and Biochemical Technology, v. 4, n.5, p. 1-7, 2013.

ANVISA. AGÊNCIA NACIONAL DE VIGILÂNCIA SANITÁRIA. Resolução da diretoria colegiada - RDC no 301, de 21 de agosto de 2019. Disponível em: <http://portal.anvisa.gov.br/> Acessado em: 31 mar. 2020.

ANVISA. AGÊNCIA NACIONAL DE VIGILÂNCIA SANITÁRIA. Resolução da diretoria colegiada- RDC no 07, de 10 de fevereiro de 2015. Disponível em: <http://bvsms.saude.gov.br/> Acessado em: 01 abr. 2020.

BENEDETTI, Natália Augusto. Avaliação da atividade antiviral dos compostos do esmalte de unha (acetato de etila e acetato de butila) no herpesvírus bovino tipo 5. 2016. Dissertação (Mestrado em Enfermagem) - Faculdade de Medicina Universidade Estadual Paulista “Júlio de Mesquita Filho", Botucatu-SP, 2016.

BRONDANI, Lara; BATISTA, Leide Dávila Rocha. Pesquisa de fungos dermatófitos queratinofílicos em amostras de areia de praças públicas do município de Porto VelhoRO. Revista Científica da Faculdade de Educação e Meio Ambiente, v. 7, n. 1, p. 137-150, 2016.

CAROBELI, Lucimara Rodrigues; DINIZ, Beatriz Vesco; CARVALHO, Nathália Martins Morette; CHINEN, Luana Yukari; TANOYE, Josiane Lie; SVIDZINSKI, Terezinha Ines Estivalet; VEIGA, Flávia Franco; NEGRI, Melissa. Fatores de virulência de fungos relacionados a zoonoses isolados em ambiente de banho e tosa de um pet shop. Revista Saúde e Meio Ambiente, v. 9, n. 2, p. 49-65, 2019. 
CHIACCHIO, Nilton; SUAREZ, Maria Victoria; MADEIRA, Celso Luis; LOUREIRO, Walter Refkalefsky. An observational and descriptive study of the epidemiology of and therapeutic approach to onychomycosis in dermatology offices in Brazil. Anais Brasileiros de Dermatologia, v. 88, n. 1, p. 3-11, 2013.

COUTINHO, Marco Aurélio da Silva; LIMA, Eziel Cardoso; GUIMARÃES, Marcio Magno Morgado; MATOS, Antônio Zilverlan Germano; ALVES, Abrãao Leal; SILVA, Rondenely Brandão; VIANA, Vicente Galber Freitas. Determinação da eficácia de diferentes solventes orgânicos na dissolução dos corantes naturais bixina e norbixina. Revista Univap, v. 22, n. 40, p. 880-886, 2017.

GARBACCIO, Juliana Ladeira; OLIVEIRA, Adriana Cristina. Biossegurança e risco ocupacional entre profissionais do segmento de beleza e estética: revisão integrativa. Revista Eletrônica de Enfermagem, v. 14, n.1, p. 702-713, 2012.

GARBACCIO, Juliana Ladeira; OLIVEIRA, Adriana Cristina. O risco oculto no segmento de estética e beleza: uma avaliação do conhecimento dos profissionais e das práticas de biossegurança nos salões de beleza. Texto e Contexto Enfermagem, v, 22, n.1, p. 989-998, 2013.

HOOG, G. S.; GUARRO, J.; GENÉ, J.; FIGUERAS, M.J. Atlas of Clinical Fungi. Amer Society for Microbiology. 9. ed., 2001.

LACAZ, Carlos da Silva; PORTO, Edward; MARTINS, José Eduardo Costa; HEINSVACCARI, Elisabeth Maria. \& TAKAHASHI DE MELO, Natalina. Tratado de Micologia médica. 9. ed. São Paulo: Sarvier, 2002.

MARTÍNEZ-HERRERA, Erick; SCHLAGER-OSPINO, Herbert; TORRES-GUERRERO, Edoardo; PORRAS-LÓPEZ, Carlos; BETANCOURT, Laura Ramos; CAMARENA, Stefanie Arroyo; ARENAS, Roberto. Frequency of mixed onychomycosis with total nail dystrophy in patients attended in a Guatemalan Dermatology Center. Revista Infectio, v. 22 , n. 2, p. 105-109, 2018.

OLIVEIRA, Flávia Márcia; DOS SANTOS, Aline Alves; ALMEIDA, Laize Santos; LIMA, Tainah Sousa Santana; MOURA, Glebson Silva; YURIKO, Simone Kameo. Adesão às Medidas de Biossegurança à Hepatite B por Manicures. Ensaios e Ciência: Ciências Biológicas, Agrárias e Saúde, v. 18, n.1, p. 83-90, 2014. 
OLIVEIRA, Maria Núbia Mendes; DOS SANTOS, Maria Solange Nogueira; ALENCAR, lolanda Gonçalves Figueiredo; LUCENA, Jalles Dantas; SOUSA, Alceu Machado; MACEDO, Francisco Nataniel Uchoa; OLIVEIRA, Thompon Lopes; FREITAS, Ana Paula Fragoso. Investigação da exposição ocupacional de manicure: um estudo transversal. Revista Internacional da Toxicologia, Risco Ambiental e Sociedade, v. 8, n.1, p. 131-175, 2015.

OLIVEIRA, Thiago Cardoso; SANTOS, Maria do Socorro Batista; SILVA, João Armindo Oliveira; BRASIL, Rogério Américo da Silva; MEDEIROS, André Henriques; SOUZA, Sônia Maria Silva. Biossegurança ações em centros de estética e embelezamento. Revista de Trabalhos Acadêmicos - Campus Niterói, v. 9, n.1, p. 1-5, 2014.

PEIXOTO, Isy; MANIQUE, Gustavo; FRANCESCONI, Valeska Albuquerque; FRANCESCONI, Fábio. Dermatofitose por Tricophyton rubrum como infecção oportunista em pacientes com doença de Cushing. Anais Brasileiro de Dermatologia, v. 85, n. 1, p. 888-890, 2010.

PEREIRA, Thaís Almeida. Avaliação da eficácia de um sistema conservante em formulações adicionadas de biomoléculas farmacêuticas e estudos de adaptação microbiana. 2011. xi, 89 f., il. Dissertação (Mestrado em Ciências da Saúde)_Universidade de Brasilia, Brasília, 2011.

RABUSKE, Camila Kaczan; BARACY, Gabriel Klafke; ORZECHOWSKI, Melissa Xavier. Avaliação de esmalte de unha como possível fômite para transmissão de dermatofitoses. 13 ${ }^{\text {a }}$ Amostra de Produção Universitária, Rio Grande do Sul/RS, 2014.

REIS, Tamara Michele; BRAIBANTE, Mara Eilsa Fortes; MIRANDA, Ana Carolina Gomes. Esmalte de unhas: uma temática para construção do conhecimento químico de funções orgânicas. Experiências em Ensino de Ciências, v.12, n.8, p. 184-196, 2017.

SAHOO, Alok Kumar; MAHAJAN, Rahul. Management of tinea corporis, tinea cruris, and tinea pedis: A comprehensive review. Indian dermatology online journal, v. 7, n. 2, p. 77-89, 2016.

SIDRIM, José Júlio Costa; ROCHA, Marcos Fábio Gadelha. Micologia Médica à luz de autores contemporâneos. Rio de Janeiro: Guanabara Koogan, 2010. 